\title{
Atherosclerosis and Antihyperlipidemic Agents
}

\author{
Laila Mahmoud Mohamed Gad* \\ Faculty of Pharmacy, King Abdulaziz University KAU, Jeddah \\ Kingdom of Saudi Arabia
}

\section{Introduction}

\subsection{Atherosclerosis \& cholesterol}

The link between cardiovascular disease (CVD) and lipids has been appreciated for a long time, but the individual role of specific lipids in predisposition to disease is constantly under question. Cardiovascular disease, including myocardial infarction (MI), heart failure, and stroke, represents the leading cause of mortality worldwide, accounting for half of the total number of deaths in the developed world (World Health Organization, 2002). CVD will result in 20.5 million deaths annually by 2020 (World Health Organization, 2002, Fig. 1), (Revkin et al. 2007).

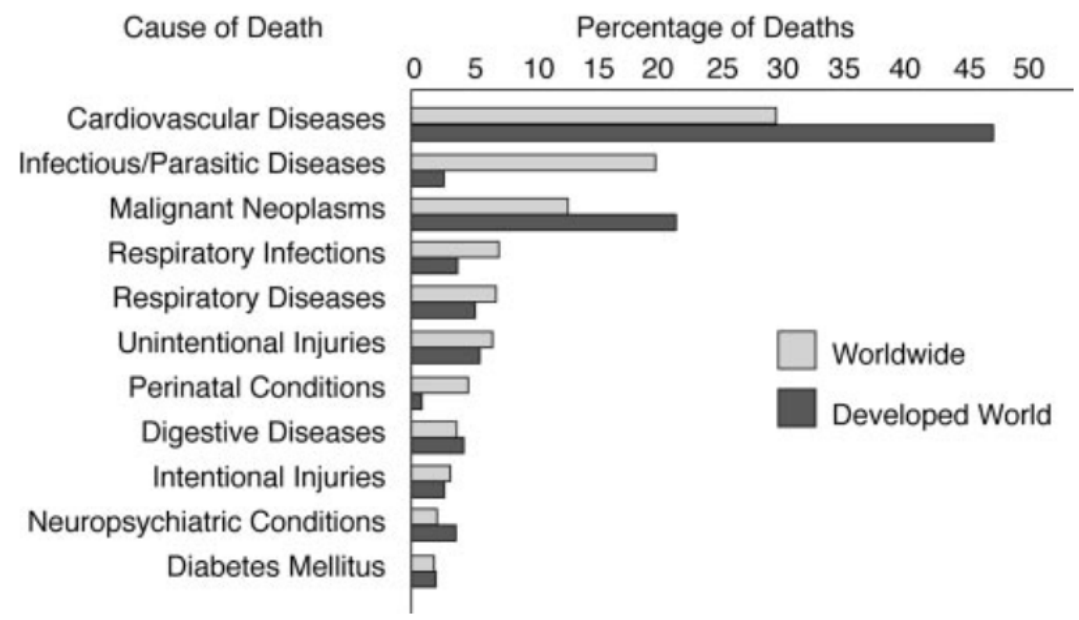

Fig. 1. Cardiovascular disease accounts for one third of deaths worldwide and half of deaths in the developed world. Data from World Health Organization (2002).

Atherosclerosis, or "athero" as we call it, is a condition underlying most cardiovascular diseases (Berliner et al. 1995) and results from an imbalance between uptake and efflux of

*Associate prof. Pharmaceutical Organic Chemistry, Faculty of Pharmacy, Mansoura University, Egypt 
cholesterol by macrophages in the wall of blood vessels. It is the progressive buildup of plaque in the arteries over time causing narrowing of the vessels and severely limiting blood flow in advanced cases. Rupture of the plaque and thrombosis may result in complete occlusion of the vessel and, ultimately, myocardial infarction or stroke (Berliner and Glass). For many of us, this plaque starts building up in our arteries in early adulthood and gets worse over time (Fig.2, for illustrative purposes).

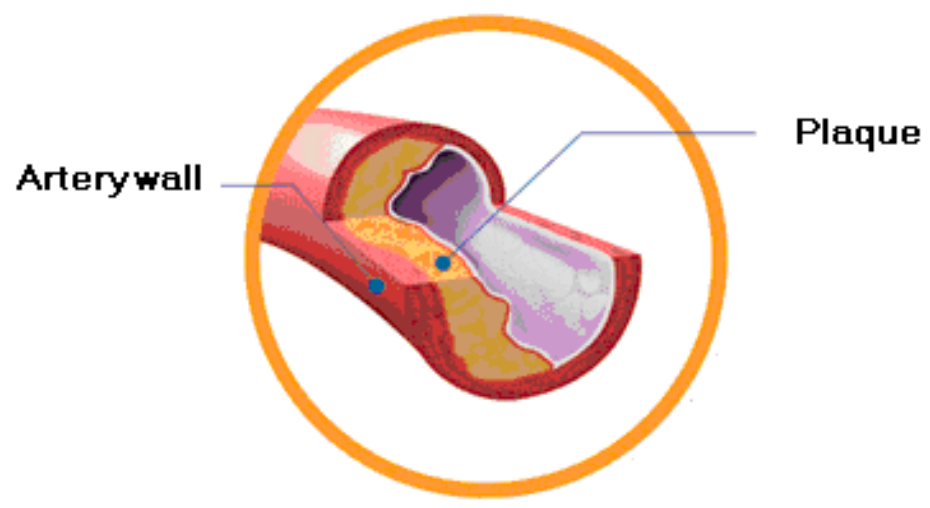

Fig. 2. Atherosclerosis; the progressive build up of plaque in arteries over time.

\section{Cholesterol}

Is a fatty substance, also called a lipid, which is produced by the liver. It is also found in foods high in saturated fat, like fatty meats, egg yolks, shellfish, and whole-milk dairy products. Since lipids are insoluble in blood, they are carried in association with proteins as lipoproteins, which allows their transport around the body. Lipoprotein complexes present in the serum have been the focus of much study in the etiology of cardiovascular disease (Goldstein, 1990).

There are five types of lipoproteins, and this classification is based on their densities. Those of lowest density, the chylomicrons, contain more lipids and less protein than those at the other extreme, high-density lipoproteins (HDLs). Between these classes are the very low-density lipoproteins (VLDLs), low-density lipoproteins (LDLs), and intermediate-density lipoproteins (IDLs). Plasma cholesterol is carried from the intestine and the liver to peripheral tissues largely in the form of LDLs. Most clinical studies concentrate on the relative abundance of HDLs and LDLs in the serum. LDL cholesterol is considered "bad" because too much of it in the blood stream can contribute to the progression of atherosclerosis, the buildup of plaque in the arteries. HDL cholesterol, on the other hand, is considered "good", it appears to be protective against cardiovascular disease (Miller, 2000), and this is generally considered to be due to the efficiency by which HDL returns cholesterol to the liver for metabolism and excretion, resulting in reduced serum cholesterol availability (Barter \& Rye, 1994).

The elevated levels of the lipoprotein (LDL) and (VLDL) are usually associated with atheroma formation. Therefore, defects in cholesterol metabolism are a major cause of cardiovascular disease, this is apparent in patients with familial hypercholesterolemia, characterized by grossly elevated levels of serum cholesterol, in particular LDL. Reduction of plasma LDL- 
cholesterol (in patients with elevated levels) is associated with a decreased incidence of coronary heart disease (CHD) (Anderson; Boden; Farnier; Fruchart and Gordon). Also, there is an increasing evidence that serum triglycerides (TGs) are strong risk factors in cardiovascular disease in many patients with normal circulating LDL-cholesterol, but with high levels of plasma TGs and concomitantly low levels of HDL-cholesterol (Cullen; Krauss and Rubins).

LDL-cholesterol has been well established as an independent risk factor since reporting of the 1948 Framingham study (Gordon et al. 1977). HDL-cholesterol is recognized as being a negative risk factor for CHD (Fruchart et al. 1998). While LDL cholesterol is a major health factor, here are a few other factors that can contribute to the progression of athero.

- Family history of early heart disease

- Diabetes

- High blood pressure

- $\quad$ Cigarette smoking

- Overweight/obesity

In a prospective follow-up study of 56 individuals with known CVD, a relationship between progression of atherosclerosis in the left main coronary artery and coronary risk factors was demonstrated (von Birgelen et al. 2004) (Fig. 3). In this study, the investigators applied three different, commonly used cardiovascular risk scores to subjects (Anderson et al. 1991 \& Assmann et al. 2002, and Conroy et al. 2003) and demonstrated a positive linear relationships between the calculated risk of CVD and plaque progression (Revkin et al. 2007).

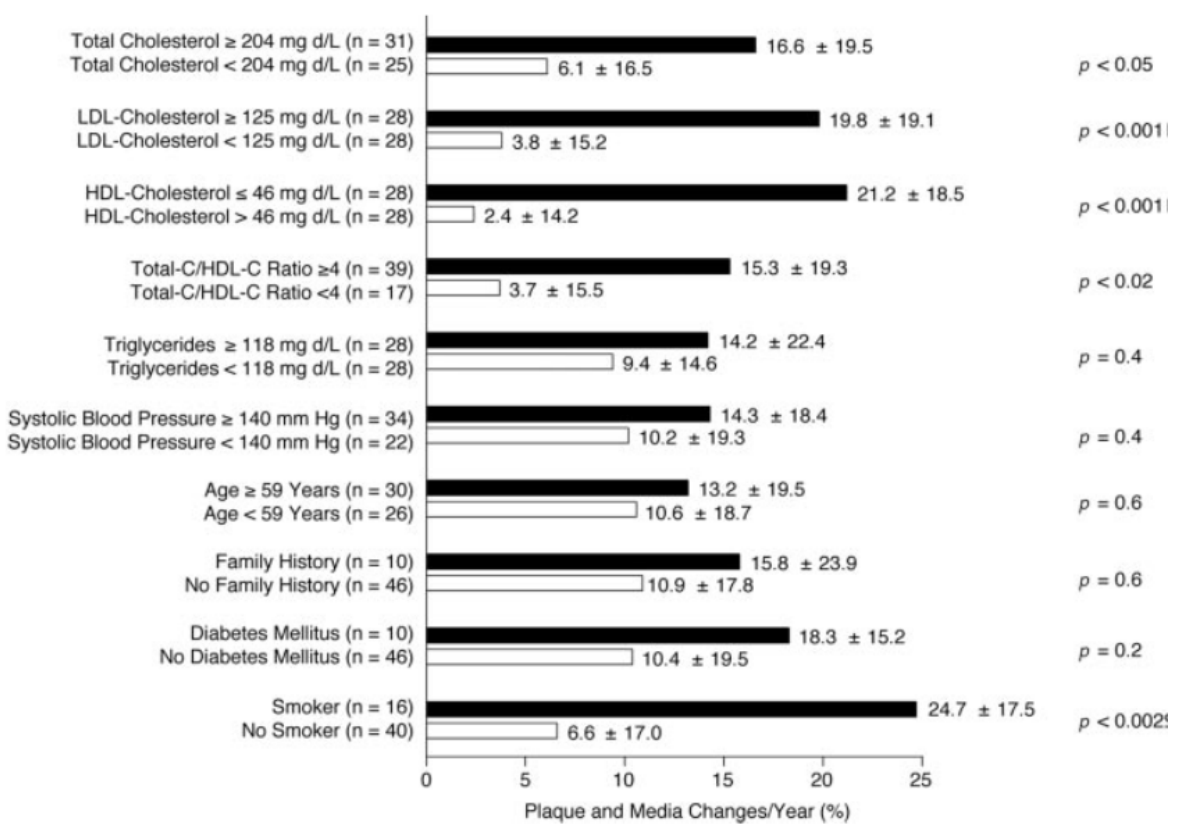

Fig. 3. Relationships between cardiovascular risk factors and atherosclerosis progression in the left main coronary artery. Data from von Birgelen et al. (2004). Reprinted from Circulation 110:1579-1585. 


\section{Biomarkers of atherosclerosis}

Atherosclerosis as a disease characterized by low-level vascular inflammation is gaining much attention recently. Some lipid parameters i.e. total serum cholesterol TC, LDL cholesterol, HDL cholesterol and triglyceride contents and also, serum markers of inflammation are considered as predictive biomarker for the prevalence of atherosclerotic disease.

Biomarkers: A biomarker is defined as a characteristic that is objectively measured and evaluated as an indicator of normal biologic or pathogenic processes or as physiologic response to a therapeutic intervention. In clinical medicine, biomarkers are routinely used in disease diagnosis, prognostication, ongoing clinical decision-making, and follow-up to assess effects of therapy.

- Commonly used biomarkers: e.g. electrocardiogram, isotopic and ultrasound imaging studies applied in multiple areas of disease management, bone densitometry in the assessment of osteoporotic fracture risk.

- Commonly used soluble biomarkers include low- and high-density lipoprotein cholesterol (LDL-C and HDL-C), triglycerides (TG), serum creatinine, and hepatocellular enzymes, as well as a host of other routine clinical laboratory measurements.

- Surrogate endpoints are biomarkers that are predictors of clinical outcomes.. Typical surrogate endpoints used to assess the clinical efficacy of cardiovascular drugs include levels of LDL-C and blood pressure.

The need for more rapid drug development highlights the role that surrogate markers may play in establishing the efficacy of drugs for managing CVD, and more specifically, atherosclerosis (Revkin et al. 2007).

\section{a. Lipid markers of atherosclerosis:}

Plasma levels of lipids and lipoproteins have been well established as strong predictors of CHD. There is, also, a stronger positive correlation of apolipoproteins (apo) with atherosclerosis and coronary events than that of the plasma lipoproteins, either cholesterol carried by lipoprotein particles or their actual concentration expressed as apo B and apo A-I.

Lipoproteins are spherical molecules that transport different amounts of cholesterol and triglycerides in the blood stream. LDL and HDL particles are rich in cholesterol, while VLDL and chylomicron particles predominantly transport triglycerides The apo B is present on the surface of LDL, VLDL and chylomicrons (one molecule at each particle), while apo AI resides on HDL particles (Fig.4). Lipoprotein (a) consist of two main components: lowdensity lipoprotein (LDL) and apolipoprotein (apo) (a) linked by a single disulphide bond between the $\mathrm{C}$ terminal of apo B100 and apo(a) kringle (K) IV type 9.The LDL particles may differ among individuals in the cholesteryl ester content of the lipid core that is surrounded by a monolayer of phospholipids, unesterified cholesterol, and apo B100. Apo (a) is made of 10 different type IV kringles (1-10) followed by kringleV and a catalytically inactive protease domain (P), (Angelo M. et al. 2008).

Conventional lipid tests determine the amount of cholesterol and triglycerides transported by all particles within the lipoprotein classes or in total plasma. Thus, cholesterol and triglycerides may be regarded as surrogate markers for their carrier-lipoprotein particles. 


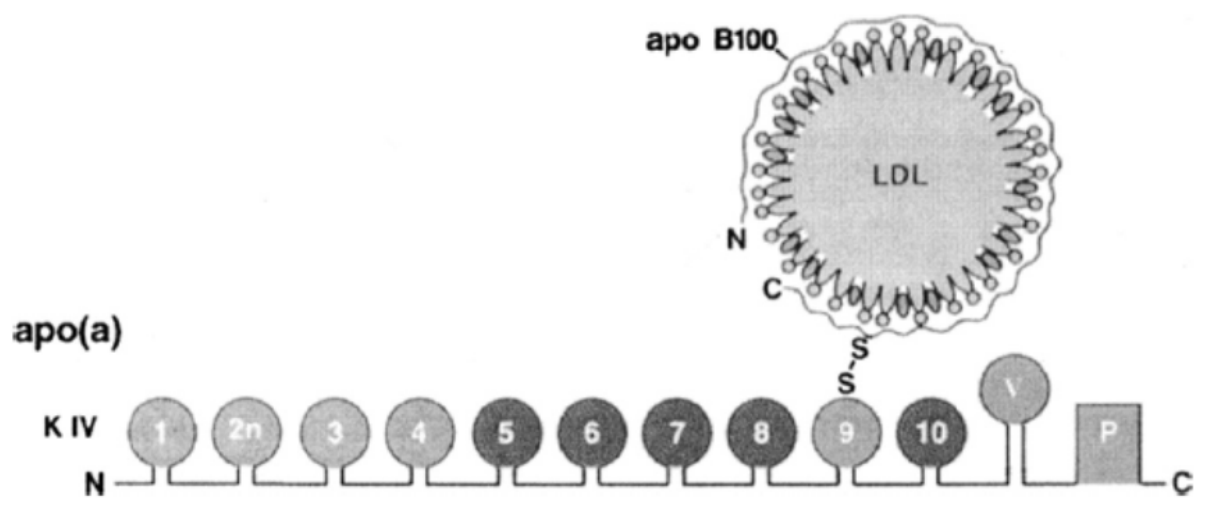

Fig. 4. Schematic representation of lipoprotein (a).

The level of apoB-containing lipoprotein particles is measured directly, mostly by immunonephelometric analysis of the plasma, on empty stomach or postprandially, whereas LDL cholesterol is mostly calculated indirectly as the difference of total cholesterol minus HDL-cholesterol and VLDL-cholesterol. In that respect, apo B can be considered a superior indicator of the global atherogenic risk over the sole quantification of LDL cholesterol and triglyceride levels, particularly in conditions of hypertriglyceridaemia characterized by high VLDL and low LDL levels. (JMB 2008, 27:148 -153).

Non-HDL cholesterol: Another approach to the measurement of atherogenic lipoproteins is the use Non-HDL cholesterol which is easily calculated by deducting the HDL cholesterol from the total cholesterol value, with no need for previous fasting of the patient. This is the cholesterol contained in VLDL and LDL particles, atherogenic triglyceride-rich lipoproteins, cholesteryl ester-enriched remnants of triglyceride-rich lipoproteins, and lipoprotein(a). Consequently, the non-HDL cholesterol is essentially the cholesterol analogue to an apo B level. The non-HDL cholesterol content is easily available within the primary screening of the lipid profile, and according to the recommendations of NCEP ATP III it is identified as a secondary therapeutic target after achieving the target levels of LDL cholesterol. (JMB, 2008, 27:148 -153).

\section{b. Circulating markers of inflammation and atherosclerosis:}

Epidemiologic and prospective studies evaluating the predictive value of the variety of circulating markers of vascular inflammation such as C-reactive protein (CRP), fibrinogen, serum amyloid A (SAA), leukocyte count in peripheral blood, immunoglobulin, adhesion molecules, cytokines and chemokines has been well established. Population studies demonstrated a moderate elevation of serum fibrinogen and leukocyte count in peripheral blood in individuals subsequently developed atherosclerotic disease. Increased fibrinogen levels were identified as strong predictors of stroke (JMB, 2008, 27:148 -153).

\section{Metabolic syndrome}

The metabolic syndrome has emerged in recent years as a major public health concern. By 2002 it was estimated that as many as $24 \%$ of American adults suffer from the metabolic 
syndrome as defined by the ATPIII criteria*).Individuals suffer from the metabolic syndrome have been demonstrated to be at increased risk of the development of hypertension, atherosclerosis, type II diabetes and cardiovascular disease. The term 'metabolic syndrome' refers to a cluster of metabolic abnormalities associated primarily with obesity including elevated plasma triglycerides (TG), low-density lipoprotein (LDLcholesterol), and very low-density lipoprotein (VLDL-cholesterol), reduced high-density lipoprotein (HDL-cholesterol), and elevations in blood pressure and fasting glucose.

\section{Dyslipidemia}

Dyslipidemia is a disruption in the amount of lipids in the blood either by increase or decrease. Most dyslipidemias are hyperlipidemias; that is, an elevation of lipids in the blood, often due to diet and lifestyle. The increased type of dyslipidemia could be differentiated as:

- Hyperlipidemia: increase in lipids; either increase in cholesterol (Hypercholesterolemia), in glycerides (Hyperglyceridemia) or in triglycerides (Hypertriglyceridemia).

- Hyperlipoproteinemia: increase in lipoproteins (usually LDL).

- Combined hyperlipidemia: that is an increase in both LDL and triglycerides.

*Executive Summary of the Third Report of the National Cholesterol Education Program (NCEP) Expert Panel on Detection, Evaluation, and Treatment of High Blood Cholesterol In Adults (Adult Treatment Panel III; ATPIII) 2001, JAMA 285: 2486-97

\section{Managing cholesterol}

The elevated levels of the lipoprotein (high cholesterol level) may depend on the lifestyle of the patient. Eating a lot of fats and not getting enough exercise can cause cholesterol levels to rise. It's also, in part, a result of the genetic makeup. Some people inherit genes associated with elevated levels of cholesterol. One type is called familial hypercholesterolemia. People with this genetic makeup can eat a healthy diet and exercise, and still have high cholesterol. Managing high cholesterol may be different for one patient to another depending on the medical history and the health of the patient. Therefore, cholesterol test, also known as a fasting lipid profile, and, along with complete medical background can work together to manage cholesterol.

\section{Cholesterol guidelines}

National Cholesterol Education Program (NCEP) guidelines recommend that all adults over age 20 have their cholesterol checked at least once every 5 years. The guidelines below give a better idea of where the cholesterol numbers of any person should be.

Total cholesterol level

- $\quad$ Less than $200 \mathrm{mg} / \mathrm{dL}$

Desirable

- $200-239 \mathrm{mg} / \mathrm{dL}$

- $240 \mathrm{mg} / \mathrm{dL}$ or higher

Borderline high

High

Total cholesterol is based on your LDL cholesterol (LDL-C) and HDL cholesterol (HDL-C) counts. Generally, a lower number is better. 
Triglycerides

- $\quad$ Less than $150 \mathrm{mg} / \mathrm{dL}$

Normal

- $150-199 \mathrm{mg} / \mathrm{dL}$

Borderline high

- $200-499 \mathrm{mg} / \mathrm{dL}$

High

- $500 \mathrm{mg} / \mathrm{dL}$ or higher

Very high

Triglycerides, like cholesterol, are another substance that can be dangerous to your health. Like LDL-C, you want to keep your triglycerides low.

LDL-C

- $\quad$ Less than $100 \mathrm{mg} / \mathrm{dL}$

Optimal

- $100-129 \mathrm{mg} / \mathrm{dL}$

- $130-159 \mathrm{mg} / \mathrm{dL}$

- $160-189 \mathrm{mg} / \mathrm{dL}$

- $190 \mathrm{mg} / \mathrm{dL}$ or higher

Near optimal / above optimal

Borderline high

High

Very high

LDL-C is considered the "bad" cholesterol because if you have too much LDL-C in your bloodstream, it can lead to the buildup of plaque in your arteries known as atherosclerosis. Generally, lower is better.

HDL-C

- $60 \mathrm{mg} / \mathrm{dL}$ or higher

High

- Less than $40 \mathrm{mg} / \mathrm{dL}$

Low

HDL-C is considered the "good" cholesterol because it helps return cholesterol to the liver, where it can be eliminated from the body. Generally, higher is better.

Source: National Cholesterol Education Program (NCEP), Webmed October 27, 2008

\section{Peroxisome proliferator-activated receptor agonists}

Peroxisome proliferator-activated receptors (PPARs) are a group of nuclear hormone receptors which function as transcription factors in the regulation of genes involved in glucose and lipid fatty acid metabolism and vessel wall function. Three PPAR subtypes have been identified: PPAR $\alpha, \gamma$ and $\delta$.

PPARa is predominantly expressed in catabolically active tissues such as liver, heart, kidney, and muscle. It is involved in the uptake and oxidation of fatty acids as well as in lipoprotein metabolism. PPAR $\gamma$ is mainly expressed in adipose tissue and regulates insulin sensitivity, glucose and fatty acid utilization as well as adipocyte differentiation (Hao Zhang et al. 2009). Recent studies have found that PPARS is also a regulator of serum lipids

There are many clinically useful drugs that produce their effects by acting on these receptors. The clinically used PPARa agonists are the fibrate class of drugs (including Fenofibrate and Gemfibrozil), which elevate HDL cholesterol levels and lower triglyceride and LDL cholesterol levels. Fibrate drugs are ligands for the fatty acid receptor PPARa (Fruchart et al. 1999). 
The clinically used PPAR $\gamma$ agonists comprise the thiazolidinedione (TZD); class of antidiabetic drugs. The thiazolidinediones (TZDs) drugs are PPARY ligands and these have beneficial effects on serum lipids in diabetic patients. The fibrate class of drugs is agonists of the PPARa isoform, and the thiazolidinediones (TZDs) that activate PPARY isoform. This is an area of great pharmaceutical interest concernining the dual $\alpha / \gamma$ agonists, which have the potential to combine the benefits of the fibrates and the TZDs, are under development (Helen V. et al. 2002).

It has been hypothesized that the combination of PPAR $\gamma$ and PPARa agonist activities in a single compound would result in synergistic improvements in insulin sensitivity and normalization of glucose metabolism as well as amelioration of the dyslipidemia associated with type 2 diabetes.

Currently marketed drugs targeting PPAR $\gamma$ like rosiglitazone used for the treatment of type 2 diabetes. We have recently reported the design and synthesis of Muraglitazar a dual PPARa $/ \gamma$ agonist which has shown excellent efficacy in animal models of type 2 diabetes and the associated dyslipidemia as well as in human clinical trial ( Fig. 5), (Xiang-Yang Ye et al. 2008).

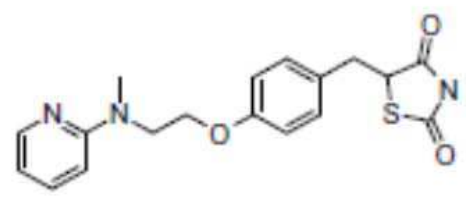

Rosiglitazone

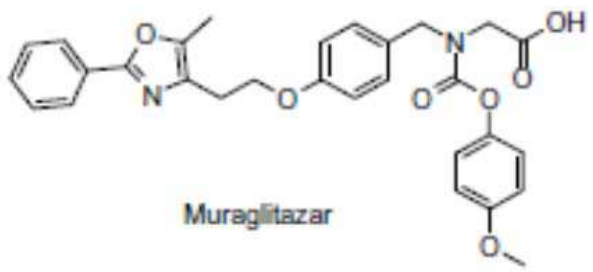<smiles>CCOC(Cc1ccc(OCCc2ccc(OS(C)(=O)=O)cc2)cc1)C(=O)O</smiles>

Tesaglitazar

Fig. 5. Structure of PPAR agonists.

\section{The antiatherosclerotic activity}

The antiatherosclerotic activity is exerted via both cholesterol lowering and direct ACAT inhibition in plaque macrophages.

Acyl-CoA: cholesterol acyl transferase (ACAT) catalyzes the intracellular formation of cholesteryl esters (CE) in all mammalian cells. It has been implicated as a key enzyme involved in cholesterol absorption, very low density lipoprotein secretion, and the formation of lipid-laden macrophages. The accumulation of CE in macrophage-derived foam cells is characteristic of the early step in the development of atherosclerosis. ACAT inhibitors reduced TC levels without affecting HDL-C. This can be attributed to decreased intestinal cholesterol absorption based on binding to bile acid (Turley SD. and Herndon MW. 1994) 


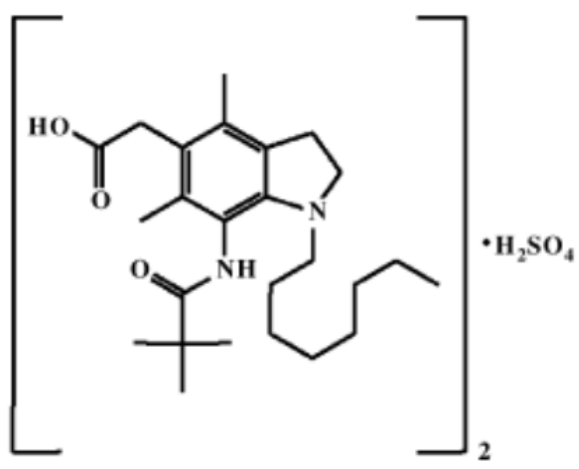

CS-505 (pactimibe sulfate)

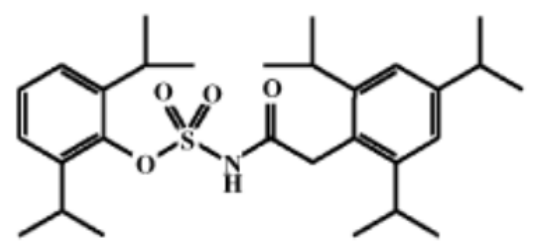

CI-1011 (avasimibe)

Fig. 6. Chemical structure of ACAT inhibitor CS-505 (pactimibe sulfate) \& CI1011(avasimibe).

Cholesterol esters (CE) are the main lipid components responsible for the development of atherosclerosis; hence they are present in the foam cells and extracellular plaque matrix, and susceptible to oxidation that could increase their atherogenic potential.

The treatment using novel ACAT inhibitor pactimibe sulfate (CS-505), avasimibe (CI-1011) (Fig.6), and a potent bile acid binding resin cholestyramine directly affects macrophages in atherosclerotic lesions. They would limit the increase in intracellular free cholesterol by its TC-lowering effect. This would allow free cholesterol to excrete into HDL, restoring the cholesterol influx/efflux balance, thus preventing foam cell formation. (Terasak N. et al. 2007).

\section{Lipid lowering drugs (LLD)}

Lipid-lowering drugs (LLD) or agents are a diverse group of pharmaceuticals that are used in the treatment of hyperlipidemia; some may lower "bad cholesterol" (LDL) more so than others, while others may prudentially increase (HDL), "the good cholesterol". Other studies showed that elevating the high-density lipoprotein (HDL), as well as, lowering the lowdensity lipoprotein (LDL) and triglyceride levels (TGs) in the serum are accepted measures in treating hyperlipidemia and atherosclerosis.

\section{Clinical trials}

The lipid lowering in hyperlipidemia is achieved clinically using statins and fibrate drugs. Since 1966, there have been 16 major trials investigating the efficacy of statin and fibrate therapy, both as a single drug treatment and as part of a multi-drug regimen (Napoli C. et al. 1997). These trials have involved the participation of some 30,000 patients, and have considered both primary and secondary prevention of coronary heart disease (CHD), myocardial infarction, stroke, and peripheral artery disease (Tylor, Faergeman, Boden). Studies by Nagao et al. (1998), involved the use of fibrate gemfibrozil to reduce cholesterol levels in hypercholesterolemia rats fed a cholesterol rich diet. 
Other studies have confirmed these results (Bruckert and Rustemeijer), while some trials involving gemfibrozil, bezafibrate, and fenofibrate indicated that fibrates can reduce LDLcholesterol to a degree comparable with that observed with statins (Feussner; Guay and Haffner). Most of the studies are of the general conclusion that statins are the drug of choice where the major dyslipidaemia is high-baseline LDL-cholesterol and that fibrates are particularly effective in the case of hypertriglyceridaemia (Farnier M. 1998). Other studies have confirmed these results while some trials involving bezafibrate, gemfibrozil, and fenofibrate indicated that fibrates can reduce LDL-cholesterol to a degree comparable with that observed with statins (Guay DR. 1999).

2. The well-established lipid lowering drugs

1. Statins: Current ATPIII guidelines for the treatment of patients with the metabolic syndrome encourage therapies that lower LDL-cholesterol and TG, and raise HDLcholesterol. Primary intervention often involves treatment with statins to improve the lipid profiles of these patients. Statins act by competitively inhibiting 3-hydroxy-3methylglutaryl-coenzyme A, (HMG-CoA) reductase, the rate-limiting enzyme in the cholesterol biosynthesis pathway in the liver (Fig.7), thus stimulates the LDL-receptors resulting in an increased clearance of LDL from the blood stream and a decrease in blood cholesterol levels. The first result can be seen after one week of use and the effect is maximal after four to six weeks. Statins are particularly well suited for lowering LDL in people at risk for cardiovascular diseases because of hypercholesterolemia .

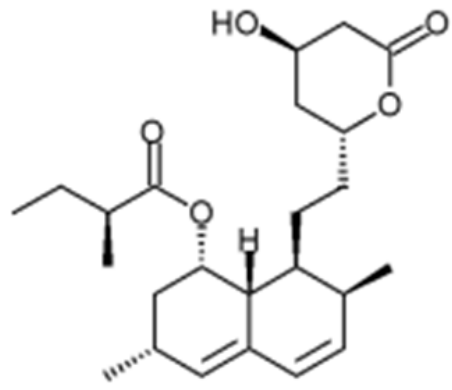

(1)

Lovastatin, the first statin to be marked

Statins are Lovastatin (1), Mevastatin -naturally-occurring compound found in red yeast rice, and Pravstatin. Statins exhibit action beyond lipid-lowering activity in the prevention of atherosclerosis; i) Improving endothelial function ii) Modulate inflammatory responses , iii) Maintain plaque stability, and iv) Prevent thrombus formation.

Rosuvastatin is a new member of the statin family with higher efficacy in reducing LDL cholesterol than other statins at comparable doses. Rosuvastatin has been shown to have an increased number of binding interactions with HMG-CoA reductase, compared to other statins. rosuvastatin also possesses a relatively long half-life and a high degree of selectivity for liver cells (the main site of cholesterol synthesis) compared with non-hepatic cells. Rosuvastatin $(10 \mathrm{mg}$ ) has been shown to improve dyslipidemia in patients with the metabolic syndrome (Naples M. et al. 2008). 


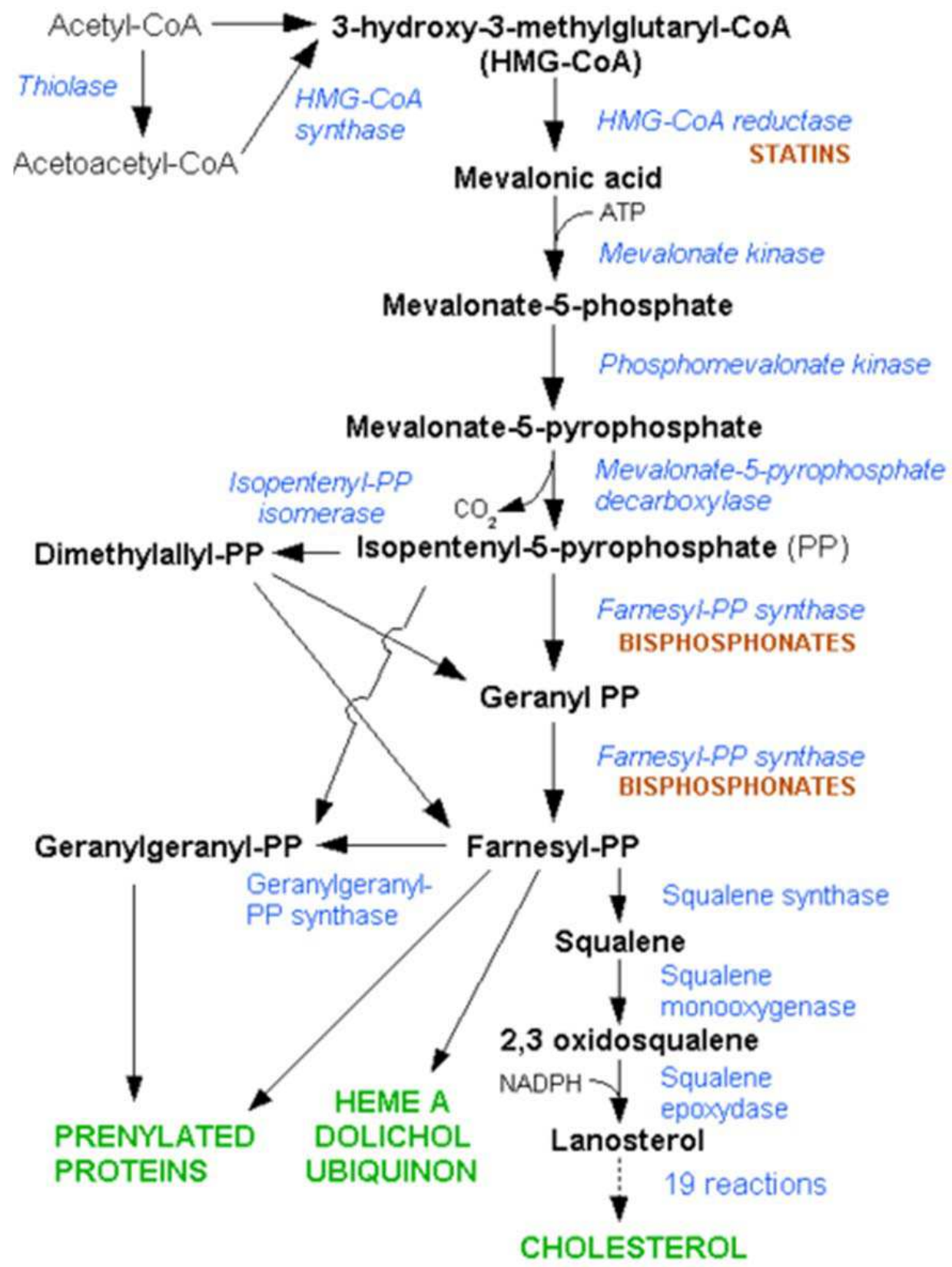

Fig. 7. Synthesis of Cholesterol through HMG-CoA reductase pathway. 
2. Fibrates: Although fibrates (e,g. clofibrate 2) are used clinically since the early 1970s, the mechanism of action of fibrates remained unelucidated until, in the 1990s, it was discovered that fibrates activate PPAR (peroxisome proliferator-activated receptors), especially PPARa. The PPARs are a class of intracellular receptors that modulate carbohydrate, fat metabolism and adipose tissue differentiation (Staels B. 1998) ; (Fruchart JC. et al. 1999). They typically lower triglyceride by $20 \%$ to $25 \%$. The newer generation fibrates: gemfibrozil (3) Bezafibrate (4) (Spieker LE. et al. 2000), and Fenofibrate (5) afford significant protection from CHD; this might be due to the agonistic effect of PPARa that inhibits inflammatory responses at the level of the vascular wall. Finally, evidence that fibrates are able to reduce levels of plasma fibrinogen, which, in turn, reduces the likelihood of thrombogenesis (Helen et.al. 2002).

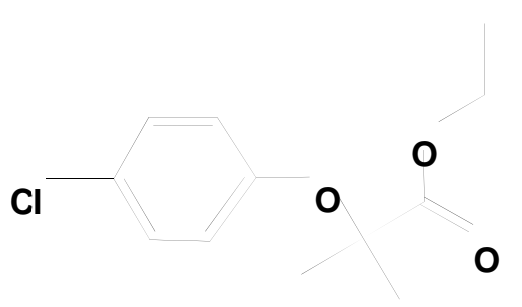

Clofibrate (2)

Ethyl 2-(4-chlorophenoxy)-2methylpropanoic acid

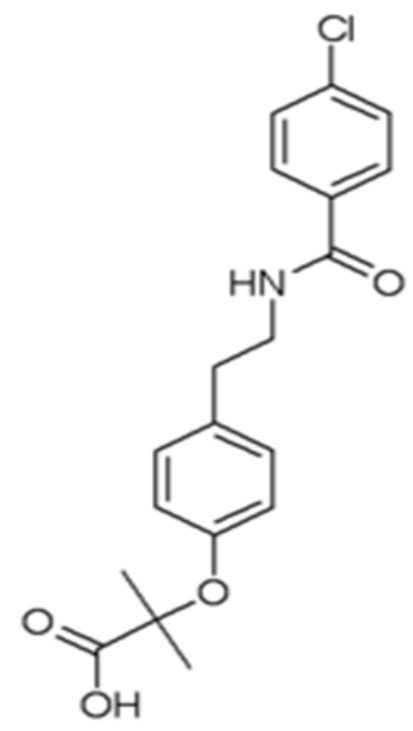

Bezafibrate (4)

2-[4-[2-[(4-chlorobenzoyl)amino] ethyl]phenoxy]-2-methylpropanoic acid

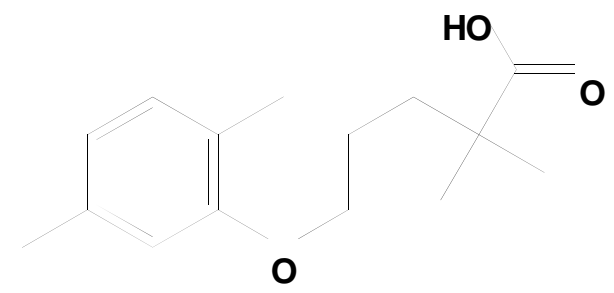

Gemfibrozil (3)

5-(2,5-dimethylphenoxy)-2,2dimethylpentatonic acid

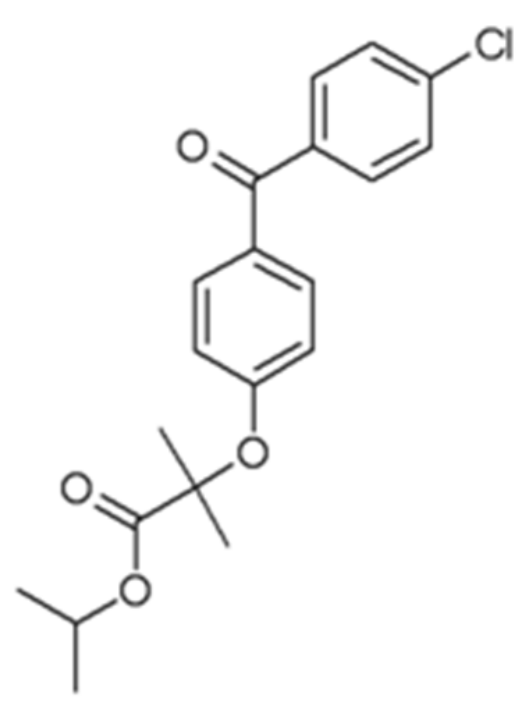

Fenofibrate (5)

Isopropyl 2-[4-(4-chlorobenzoyl) phenoxy]2-methyl propanoate 
3. Niacin: 3-pyridinecarboxylic acid . also know as nicotinic acid or vitamin B3, the name niacin was derived from nicotinic acid + vitamin, it is also referred to as "vitamin PP", a name derived from the absolute term "pellagra-preventing factor". Over the years, niacin has gained recognition as an atheroprotective agent, in part because of its capacity to lower the plasma levels of cholesterol, triglycerides by $20-50 \%$, and verylow- and low-density lipoproteins. It may lower LDL by $5-25 \%$ and to substantially raise high-density lipoprotein. by $15-33 \%$..In high doses, niacin has also been reported to lower the plasma level of lipoprotein(a) (Angelo M. 2008).

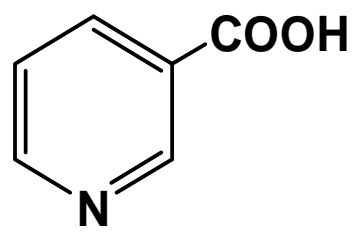

Niacin (6)

3-pyridinecarboxylic acid

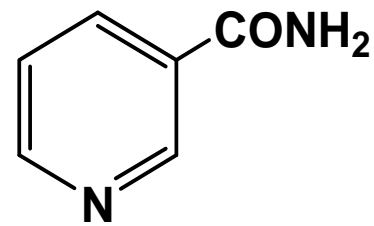

Nicotinamide (7)

3-pyridinecarboxamide

4. Ezetimibe: (Zetia) is a selective inhibitor of dietary cholesterol absorption. In addition to this decreased cholesterol absorption leads to an increase in LDL-cholesterol uptake into cells, thus decreasing levels in the blood plasma (Rossi S, 2006).

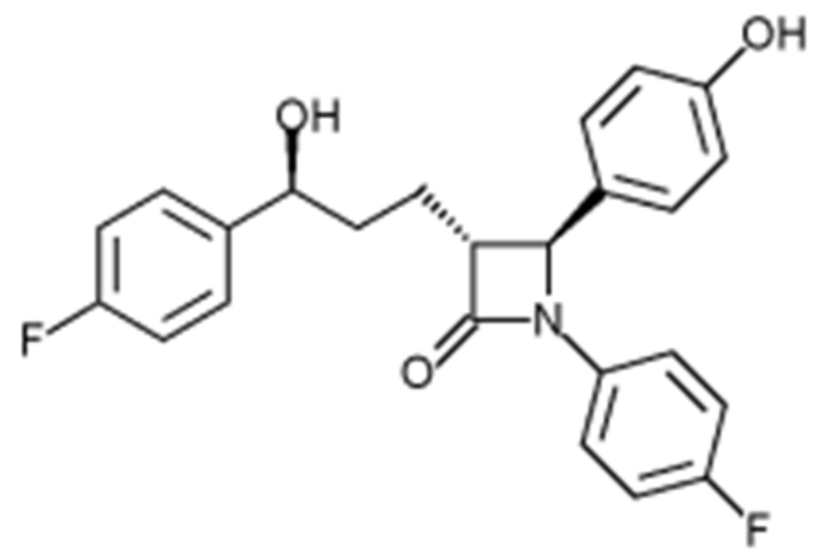

Ezetimibe (8)

(3R, 4S)-1-(4-fluorophenyl)-3- ((3S)-3-(4-fluorophenyl)-

3-hydroxypropyl)-4-(4-hydroxyphenyl)-2-azetidinone

5. Bile acid sequestrants: e.g. Colesevelam hydrochloride (WelChol -- Sankyo) is a nonabsorbed, polymeric, lipid-lowering agent that binds with bile acids in the intestine and significantly reduces their reabsorption. As the bile acid pool becomes depleted, there is an increased conversion of cholesterol to bile acids, thereby reducing cholesterol concentrations. The mechanism of action of colesevelam is similar to that of cholestyramine (e.g., Questran) and colestipol (Colestid). However, the new drug has a greater binding affinity for bile acids, permitting the use of a lower dosage, and appears to have a lower incidence of gastrointestinal (GI) adverse events and a lower potential 
for drug interactions. Colesevelam is indicated for use, alone or in combination with a hydroxymethyl-glutaryl-coenzyme A (HMG-CoA) reductase inhibitor (a "statin"), as adjunctive therapy to diet and exercise for the reduction of elevated low-density lipoprotein cholesterol (LDL-C) in patients with primary hypercholesterolemia (Fredrickson Type IIa). In the clinical studies, colesevelam reduced LDL-C concentrations by $15 \%$ to $18 \%$, and increased high-density lipoprotein cholesterol (HDL-C) concentrations by 3\%. There were small increases in triglyceride concentrations, but these were not statistically different from the results in those receiving placebo.

6. Phytosterols: have cholesterol-lowering properties; reducing cholesterol absorption in intestines, and may act in cancer prevention. Phytosterols naturally occur in small amount in vegetable oils, especially soybean oil. Sterols can reduce cholesterol in human subjects by up to $15 \%$ (Rossi S, 2006).

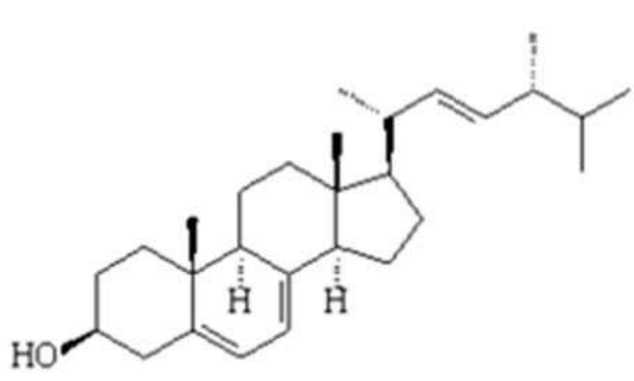

Ergosterol (9)

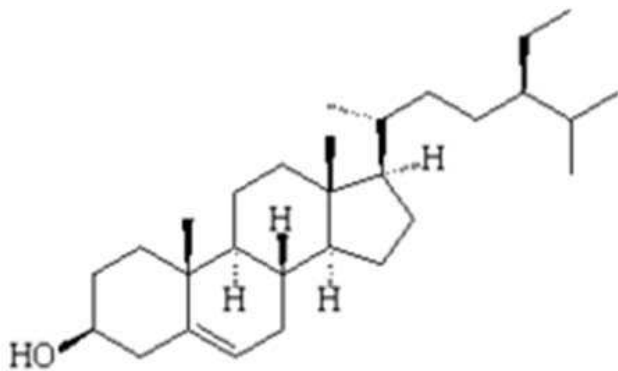

$\beta$-Sitosterol (10)

7. Thiazolidinediones: TZDs are now seen to fight several aspects of metabolic disease within Type II diabetes. They reduce blood glucose while enhancing insulin sensitivity, and also have a profound effect on lipid metabolism. Patients with Type II diabetes commonly present with elevated plasma levels of TGs and low HDLs. TZDs increase TG lipolysis in VLDLs, reducing the level of TGs and increasing HDLs. TZDs increase TG lipolysis in VLDLs, reducing the level of TGs and increasing HDLs and have also been shown to inhibit the progression of atherosclerosis in animal models (Schoonjans \& Auwerx, 2000).

The mechanism of thiazolidinediones (TZDs) drugs by which the change in serum lipid profile occurs is not clear, but may involve increases in serum lipoprotein lipase and increased lipid uptake by adipocytes and skeletal muscle (Burant and Park). It is also possible that it results from direct effects of the drug acting on PPARa (Smith, 2001). It is not clear, especially in humans, if PPARY promotes lipid clearance in the liver in a manner similar to PPARa.

\section{Synthetic hypolipidemic agents}

A continued research effort has been underway over the past several years in the area of the development of hypolipidemic agents (Chapman JM. et al. 1979\&,1983 \& Wyrick SD. et al. 1984). 
Chapman and co-workers initially reported the hypolipidemic activity of phthalimides and N-substituted phthalimides including alkyl, methyl ketone, carboxylic acids, and acetate esters of varying chain length were synthesized and tested for hypolipidemic activity in mice, compounds (1), (2), and (3) were found to afford the most significant reduction in serum cholesterol and triglyceride levels. Phthalimides, the parent compound, decreased serum cholesterol and triglyceride levels by $43 \%$ and $56 \%$ respectively, in mice after 16 days of dosing at an optimum dose of 20mg/ kg/ day (Chapman JM. et al. 1979)

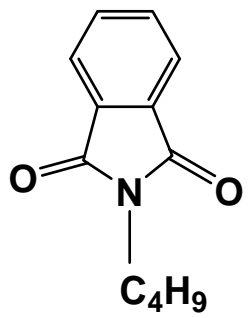

(1)

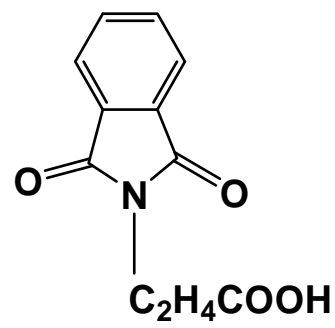

(2)

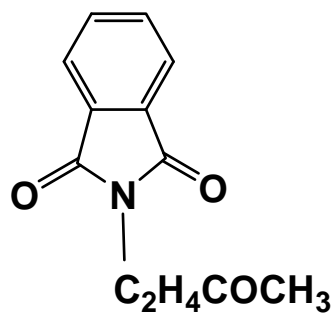

(3)

The hypolipidemic effects of aromatic versus non aromatic imides were investigated. Thus, a number of $\mathrm{N}$-substituted phthalimides (4),1,8-naphthalimide (5), succinimide (6), and glutarimide (7) derivatives demonstrated significant hypolipidemic activity at 20 $\mathrm{mg} / \mathrm{kg} /$ day in male mice $(\sim 25 \mathrm{gm})$ after16 days of intrapretonially dosing. The dose of 20 $\mathrm{mg} / \mathrm{kg} /$ day was selected for structure activity relationship SAR study because this dose proved to be the optimum dose when testing phthalimides and 3-N-(1,8naphthalimide)propionic acid. Most of the derivatives at $20 \mathrm{mg} / \mathrm{kg} /$ day demonstrated improved activity over clofibrate at $150 \mathrm{mg} / \mathrm{kg} /$ day. In general, 1,8-naphthalimide and glutarimide derivatives appeared to be less active than phthalimides and succinimide. Removal of phenyl ring of phthalimides resulting in succinimide led to less hypolipidemic activity in general. The loss of aromatic system of 1,8-naphthalimide resulting in glutarimide led to a slight loss of anticholesterolemic activity and only a-phenylglutarimide show slight improvement of antitriglyceridemic activity (Chapman JM. et al. 1984).

The importance of rigid imide ring system for hypolipidemic activity was determined. Therefore, two series of cyclic imides; diphenimide (8) and its open acyclic imides; dibenzimide (9), and succimide (10), and diacetimide (11) were examined for hypolipidemic activity in mice. 


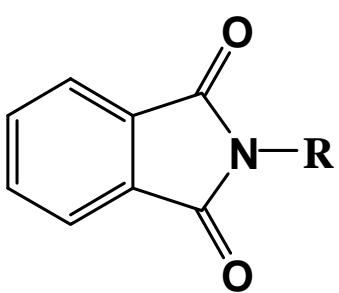

(4)

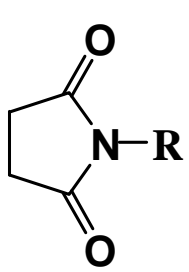

(6)

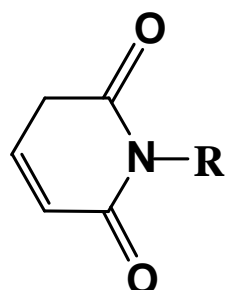

(7)

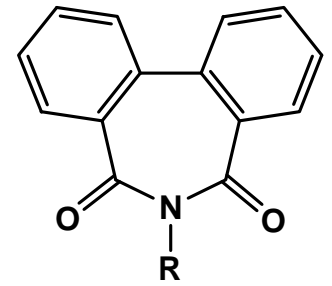

(8)<smiles>CN1C(=O)CCC1=O</smiles>

$\mathbf{R}$

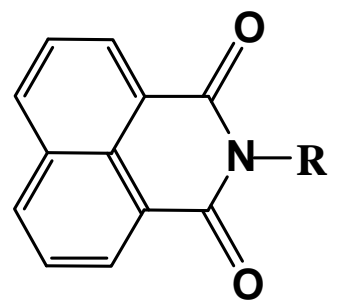

(5)

$$
\begin{aligned}
& \mathbf{R}= \mathrm{H} \\
& \mathbf{n}-\mathrm{C}_{4} \mathrm{H}_{9} \\
& \mathrm{n}-\mathrm{C}_{5} \mathrm{H}_{11} \\
& \mathrm{CH} \mathrm{H}_{2} \mathrm{CH}_{2}-\mathrm{COOH} \\
& \mathrm{CH}_{2} \mathrm{CH}_{2}-\mathrm{CO}-\mathrm{CH}_{3}
\end{aligned}
$$<smiles>CP(C(=O)c1ccccc1)N(P)C(=O)c1ccccc1</smiles>

(9)<smiles>CC(=O)N(C)C(C)=O</smiles>

$\mathbf{R}$

(11)

(10)

\section{$\mathrm{R}=\mathrm{C}_{4} \mathrm{H}_{9}, \mathrm{CH}_{2} \mathrm{CH}_{2} \mathrm{COCH}_{3}, \mathrm{CH}_{2} \mathrm{CH}_{2} \mathrm{COOH}, \mathrm{CH}_{2} \mathrm{CH}_{2} \mathrm{COOC}_{2} \mathrm{H}_{5}$}

It was shown that the rigid imide ring system was not necessary for hypercholesterolemia activity. However, the cyclic imide's structure was a necessary requirement for a good hypotriglyceridemic activity (Voorstad. et.al. 1985).

A series of nitrogen substituted N-butan-3-one derivatives of cyclic imides; such as phthalimides (12), o-benzosulphimide (13), 1,8-naphthalimide (14), and diphinimide (15) were prepared (Chapman JM. et al. 1990). 
<smiles>[R]c1ccc2c(c1)C(=O)N(CCC([X])C)C2=O</smiles><smiles>[R]c1ccc2c(c1)S(=O)(=O)N(CCC([X])C)C2=O</smiles>

$$
\mathrm{R}=\mathrm{H}, \mathrm{Cl}, \mathrm{CH}_{3}, \mathrm{NO}_{2}
$$
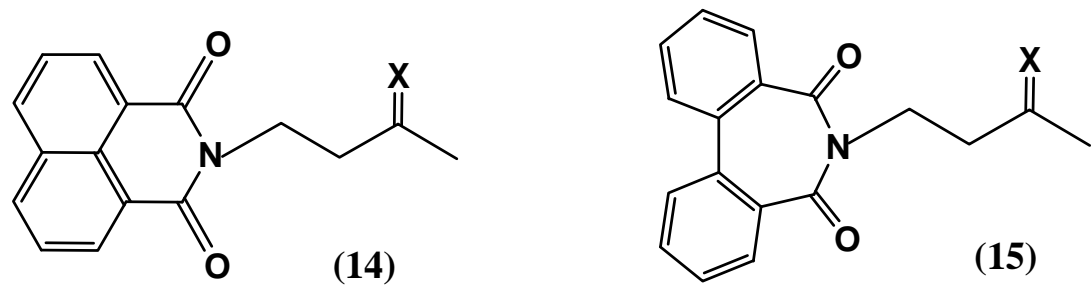

\section{$\mathrm{X}=\mathrm{a}) \mathrm{NNH}-\mathrm{CO}-\mathrm{NH}_{2}, \quad$ b) $\mathrm{NNH}-\mathrm{CS}-\mathrm{NH}_{2}, \quad$ c) $\mathrm{NNH}-\mathrm{CO}-\mathrm{CH}_{3}$}

Molecular modeling programs were used to compare the 3D structures of the some derivatives of some cyclic imides with the already known Clofibrate (2) (ethyl ester of 2,2dimethylphenoxyacetic acid), These overlays were made with the aid of computer Accelry's soft wares at the Computer laboratory, Faculty of Pharmacy, KAU, KSA.

The Changes in the structure of phthalimide involving the imide's ring system led to the preparation of new compounds with potent and significant antihyperlipidemic effect; this is due to the good efficacy of their binding to the fatty acid receptor sites (Hall IH et al. 1986).

The promising hypolipidemic activity of certain cyclic imides derivatives in experimental animals strongly potentiate and recommend more researches in this area (El-Zahabi MA et al. 2010). Moreover; the newly synthesized compounds may be useful in the prevention of cardiovascular disease in a growing population suffering from lifestyle-induced metabolic dysfunction (Project 040/1428 H, Gad L M. et al.;funded by the Deanship of Scientific Research, King Abdulaziz University, KAU, Jeddah, KSA).

\section{References}

[1] Anderson KM, Castelli WP and Levy D (1987). Cholesterol and mortality. 30 years of follow-up from the Framingham study. JAMA 257: 2176-2180. Abstract-MEDLINE

[2] Anderson KM, Wilson PW, Odell PM and Kannel WB (1991). An updated coronary risk profile. A statement for health professionals. Circulation 83: 356-362

[3] Assmann Gerd, Paul Cullen, Helmut Schulte (2002). Clinical Investigation and Reports, Simple Scoring Scheme for Calculating the Risk of Acute Coronary Events Based on the 10-Year Follow-Up of the Prospective Cardiovascular Münster (PROCAM) Study . Circulation 105:310

[4] Barter PJ and Rye KA (1994). High-density lipoproteins and coronary heart disease. J Cardiovasc Risk 1: 217-221. 
[5] Berliner JA, Navab M, Fogelman AM, Frank JS, Demer LL, Edwards PA, Watson AD and Lusis AJ (1995). Atherosclerosis: basic mechanisms. Oxidation, inflammation, and genetics. Circulation 91:2488-2496.

[6] von Birgelen C, Hartmann M, Mintz GS, et al. (2004) Relationship between cardiovascular risk as predicted by established risk scores versus plaque progression as measured by serial intravascular ultrasound in left main coronary arteries. Circulation 110:1579-1585

[7] Boden WE (2000). High-density lipoprotein cholesterol as an independent risk factor in cardiovascular disease: assessing the data from Framingham to the Veterans Affairs high-density lipoprotein intervention trial. Am J Cardiol 86: 19L-22L. Abstract MEDLINE

[8] Barooah N, Tamuly C, Baruah J. (2005) Synthesis, characterisation of few N-substituted 1,8-naphthalimide derivatives and their copper(II) complexes J. Chem. Soc. 117(2):117.

[9] Chapman JM, Cocolas GH, and Hall IH (1979) Hyperlipidemia activity of phthalimides derivatives. 1. N-Substituted Phthalimide derivatives. J. Med. Chem. 22: 1399.

[10] Chapman JM, Voorstad PJ, Cocolas GH, and Hall IH (1983). Hypolipidemic activity of phthalimide derivatives. 2. N-Phenylphthalimide derivatives. J. Med. Chem. 28: 237243

[11] Chapman JM, Cocolas GH, and Hall IH (1983). Hypolipidemic activity of phthalimide derivatives, IV: Further chemical modification and investigation of the hypolipidemic activity of N-substituted imides. J. Pharm. Sci. 72: 1344-7

[12] Chapman JM, Wyrick SD, Maguire JH, Cocolas GH, and Hall IH (1984). Hypolipidemic Agents of Phthalimide Derivatives, 6. Effect of Aromatic vs. Non-Aromatic Imides. J. Pharm. Res. 6: 267-269

[13] Chapman JM, DeLucy P, Wong OT, and Hall IH (1990). Structure Activity Relationship of Imido N-Alkyl Semicarbazones, Thiosemicarbazones and Acetyl hydrazones as Hypolipidemic Agents in Rodents. J. Lipid. 25, No.7: 391-397.

[14] Chapman JM, Wyrick SD, Voorstad PJ, Maquire JH, Cocolas GH, and Hall IH (1984). Hypolipidemic activity of phthalimide derivatives V: Reduced and hydrolytic products of simple cyclic imides. J. Pharm. Sci. 73,(10):1482-4.

[15] Conroy RM, Pyo"ra"la“ K, Fitzgerald A et al. (2003). Estimation of ten-year risk of fatal cardiovascular disease in Europe: the SCORE project. Eur Heart j; 24:987-1003.

[16] The Coronary Drug Project Research Group. Clofibrate and niacin in coronary heart disease. JAMA (1975) 231: 360 -381.

[17] Cullen P (2000). Evidence that triglycerides are an independent coronary heart disease risk factor. Am J Cardiol 86: 943-949.

[18] El-Zahabi MA, Gad LM, Bamanie FH, and Al-Marzooki Z (2010). Synthesis of new of Cyclic Imides derivatives with potential Hypolipidemic Activity.(project 048/1426H; funded by the Deanship of Scientific Research, King Abdulaziz University, KAU) Available online 25/11/2010, Med Chem Res (2012) 21, issue (1):75-84.

[19] Faergeman O (2000). Hypertriglyceridemia and the fibrate trials. Curr. Opin. Lipidol 11: 609-614 
[20] Farnier M (1998). Cerivastatin in the treatment of mixed hyperlipidemia: the RIGHT study. The Cerivastatin Study Group. Cerivastatin, Gemfibrozil, Hyperlipidemia Treatment. Am J Cardiol 82: 47J-51J.

[21] Feussner G, Kurth B and Lohrmann J. (1997) Comparative effects of bezafibrate and micronised fenofibrate in patients with type III hyperlipoproteinemia. Eur. J. Med. Res. 2: pp. 165-168. Abstract-MEDLINE

[22] Fruchart JC, Brewer HB and Leitersdorf E (1998). Consensus for the use of fibrates in the treatment of dyslipoproteinemia and coronary heart disease. Fibrate Consensus Group. Am J Cardiol 81: 912-917. Abstract-MEDLINE

[23] Fruchart JC, Duriez P and Staels B (1999). Molecular mechanism of action of the fibrates (French). J Soc Biol 193: 67-75. Abstract-MEDLINE

[24] Gad LM., El-Zahabi MA, Ginienah M M., Bamanie F H, Ghareib S A. and Al-Marzooki Z H.(2011). Synthesis of new 1,8-naphthalimide derivatives with potential antihyperlipidemic activities.(Under publication).

[25] Goldstein JL (1990). Familial hypercholesterolemia, In: Scriver CR, Beaudet AI, Sly WS and Valle D, Editors. The Metabolic Basis of Inherited Diseases. McGraw-Hill, New York, 1215-1250.

[26] Gordon T, Castelli WP, Hjortland MC, Kannel WB and Dawber TR (1977). High density lipoprotein as a protective factor against coronary heart disease. The Framingham Study. Am J Med 62: 707-714.

[27] Guay DR (1999). Micronized Fenofibrate: a new fibric acid hypolipidemic agent. Ann Pharmacother. 33: 1083-1103

[28] Haffner SM. (2000). Coronary heart disease in patients with diabetes. N Engl J Med.; 342: 1040-1042

[29] Hall IH, Murthy ARK, and Wyrick SD (1986). Hypolipidemic Activity of 6-Substituted 6,7-Dihydro-5H-dibenz[c,e]azepine and the Effects of 6,7-Dihydro-5Hdibenz[c,e]azepine on Lipid Metabolism of Rodents. J. Pharm Sci. 75, No.6: 622-626

[30] Helen Vosper, Guennadi A. Khoudoli, Tracey L. Graham and Colin N. A. (2002). Peroxisome proliferator-activated receptor agonists, hyperlipidaemia, and atherosclerosis. pharmacology \& therapeutic 95, Issue 1: 47-62.

[31] Krauss RM, (1998). Atherogenicity of triglyceride-rich lipoproteins. Am J Cardiol 81: 13B-17B

[32] Larsen SD.and Spilman CH (1993). New Potential Therapies for the Treatment of atherosclerosis. Ann. Rep. Med. Chem. 28: 217-26.

[33] Miller M (2000). Differentiating the effects of raising low levels of high-density lipoprotein cholesterol versus lowering normal triglycerides insights from the veterans affairs high-density lipoprotein intervention trial. Am J Cardiol 86: 23-27.

[34] Murthy AR, Wyrick SD, Voorstad PJ, and Hall IH (1985). Hypolipidemic activity of Nsubstituted diphenimides in rodents. Eur. J. Med. Chem. 20, No.6: 547-550

[35] Napoli C, Lepore S, Chiariello P, et al. (1997) Longterm treatment with pravastatin alone and in combination with gemfibrozil in familial type IIB hyperlipoproteinemia or combined hyperlipidemia. J Cardiovasc. Pharmacol Ther. 2(1):17-26. 
[36] Revkin JH, Shear CL, Pouleur HG, Ryder SW, and Orloff DG (2007). Biomarkers in the Prevention and Treatment of Atherosclerosis: Need, Validation, and Future. Pharmacol Rev 59:40-53

[37] Rossi S; editor. Australian Medicines Handbook, (2006), ISBN 0-9757919-2-3 through Wikipedia, the free encyclopedia, ONLINE.

[38] Rubins HB (2000). Triglycerides and coronary heart disease: implications of recent clinical trials. J Cardiovasc Risk 7:339-345. Abstract-MEDLINE

[39] Rustemeijer C, Schouten JA, Voerman HJ, Hensgens HE, Donker AJ and Heine RJ (2000). Pravastatin compared to bezafibrate in the treatment of dyslipidemia in insulin-treated patients with type 2 diabetes mellitus. Diabetes Metab Res Rev 16: 8287. Abstract-MEDLINE

[40] Spieker LE, Noll G, Hannak M, and Luscher TF (2000). Efficacy and tolerability of fluvastatin and bezafibrate in patients with hyperlipidemia and persistently high triglyceride levels. J. Cardiovasc Pharmacol, 35: 361-365.

[41] Sprecher DL (2000). Raising high-density lipoprotein cholesterol with niacin and fibrates: a comparative review. Am J Cardiol 86: 46-50.

[42] Staels B, Dallongeville J, Auwerz J, Schoonjans K, Leitersdorf E, Fruchart J, (1998).Mechanism of action of fibrates on lipid and lipoprotein metabolism. Circulation. 98, issue 19: 2088-2093

[43] Terasaka N , Miyazaki A, Kasanuki N, Ito K, Ubukata N, Koieyama T, Kitayama K, Tanimoto T, Maeda N, Inaba T (2007). ACAT inhibitor pactimibe sulfate (CS-505) reduces and stabilizes atherosclerotic lesions by cholesterol-lowering and direct effects in apolipoprotein E-deficient mice. Atherosclerosis 190: 239-247

[44] Turley SD, Herndon MW, Dietschy JM (1994). Reevaluation and application of the dualisotope plasma ratio method for the measurement of intestinal cholesterol absorption in the hamster. J Lipid Res. 35: 328-39.

[45] Tylor HA (1987). Lowering plasma cholesterol levels decrease risk of coronary artery disease: An overview of clinical trials in Hypercholesterolemia

[46] Voorstad PJ, Chapman JM, Cocolas GH, Wyrick SD, and Hall IH (1985). Comparison of the Hypolipidemic Activity of Cyclic vs. AcyclicImides. J. Med.Chem. 28: 9-12

[47] Wyrick SD, Voorstad PJ, Cocolas GH, and Hall IH (1984). Hypolipidemic Activity of Phthalimide Derivatives. 7. Structure-Activity Studies of Indazolone Analogues. J. Med. Chem. 27: 768-772.

[48] Xiang-Yang Ye a, et al., (2008), Design, synthesis, and structure-activity relationships of piperidine and dehydropiperidine carboxylic acids as novel potent dual PPAR $a / \gamma$ agonists. Bioorganic \& Medicinal Chemistry Letters 18: 3545-3550

[49] Zhang H et al. (2009). Design, synthesis and structure-activity relationships of azole acids as novel, potent dual PPAR a/ $\gamma$ agonists. Bioorg \& Med Chem Lett. 19: 14511456.

[50] J. Medicinal Biology; 2008, JMB 27:148 -153, (Review article). 


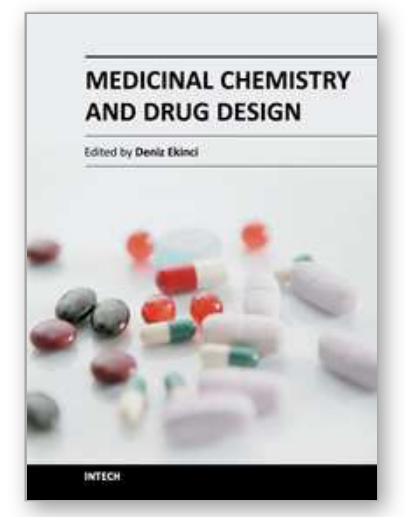

\author{
Medicinal Chemistry and Drug Design \\ Edited by Prof. Deniz Ekinci
}

ISBN 978-953-51-0513-8

Hard cover, 406 pages

Publisher InTech

Published online 16, May, 2012

Published in print edition May, 2012

Over the recent years, medicinal chemistry has become responsible for explaining interactions of chemical molecules processes such that many scientists in the life sciences from agronomy to medicine are engaged in medicinal research. This book contains an overview focusing on the research area of enzyme inhibitors, molecular aspects of drug metabolism, organic synthesis, prodrug synthesis, in silico studies and chemical compounds used in relevant approaches. The book deals with basic issues and some of the recent developments in medicinal chemistry and drug design. Particular emphasis is devoted to both theoretical and experimental aspect of modern drug design. The primary target audience for the book includes students, researchers, biologists, chemists, chemical engineers and professionals who are interested in associated areas. The textbook is written by international scientists with expertise in chemistry, protein biochemistry, enzymology, molecular biology and genetics many of which are active in biochemical and biomedical research. We hope that the textbook will enhance the knowledge of scientists in the complexities of some medicinal approaches; it will stimulate both professionals and students to dedicate part of their future research in understanding relevant mechanisms and applications of medicinal chemistry and drug design.

\title{
How to reference
}

In order to correctly reference this scholarly work, feel free to copy and paste the following:

Laila Mahmoud Mohamed Gad (2012). Atherosclerosis and Antihyperlipidemic Agents, Medicinal Chemistry and Drug Design, Prof. Deniz Ekinci (Ed.), ISBN: 978-953-51-0513-8, InTech, Available from: http://www.intechopen.com/books/medicinal-chemistry-and-drug-design/atherosclerosis-andantihyperlipidemic-agents

\section{INTECH}

open science / open minds

\section{InTech Europe}

University Campus STeP Ri

Slavka Krautzeka 83/A

51000 Rijeka, Croatia

Phone: +385 (51) 770447

Fax: +385 (51) 686166

www.intechopen.com

\section{InTech China}

Unit 405, Office Block, Hotel Equatorial Shanghai

No.65, Yan An Road (West), Shanghai, 200040, China

中国上海市延安西路65号上海国际贵都大饭店办公楼405单元

Phone: +86-21-62489820

Fax: +86-21-62489821 
(C) 2012 The Author(s). Licensee IntechOpen. This is an open access article distributed under the terms of the Creative Commons Attribution 3.0 License, which permits unrestricted use, distribution, and reproduction in any medium, provided the original work is properly cited. 